\title{
Distribution and gene expression of neuropeptides during brain development
}

\author{
W. Sienkiewicz \\ University of Warmia and Mazury, Faculty of Veterinary Medicine, Department of Functional \\ Morphology, Division of Animal Anatomy, 10-719 Olsztyn, Oczapowskiego str 13, Poland
}

Neuropeptides and catecholamines are biologically active substances which play the roles of neurotransmitters, neuromodulators and cotransmitters in the central nervous system (CNS). These substances are known to regulate and influence a wide spectrum of functions; such as food and water intake, thermoregulation, growth and maturation, sexual behaviour, reproduction, function of the hypothalamic-hypophysial-organ axes, and many others. This large family of neuropeptides is comprised of around 40 fundamental polypeptides, which does not include their derivatives and related peptides. Localization and development of several of them was intensively studied in the brain of a wide range of species, including the rat and the pig. In this present paper, data regarding distribution of catecholamine synthesising enzymes and some neuropeptides, as well as their gene expression during development in the rodent and porcine brain are summarised and related to development of $\mathrm{LHRH}$-containing structures.

\section{Introduction}

The number of putative neurotransmitters has dramatically increased during recent decades. In addition to the classical neurotransmitters and a few amino acids, at least 40 neuropeptides have been found: all these substances can act as chemical messengers in the mammalian nervous system. Neuropeptides are divided into several families, such as the vasoactive intestinal peptide (VIP)-glucagon family, neuropeptide Y (NPY) and related peptides, tachykinins, hypothalamic hormones, hypothalamic releasing and inhibiting hormones, a large group of "miscellaneous" peptides (including galanin), and some "novel" neuropeptides. Immunohistochemistry and molecular biology methods have allowed the precise localization of these neurotransmitters and neuropeptides, and thus, contributed to the understanding of how certain regions of the brain function. This expanding body of knowledge of the relationship between structure and function has been accompanied by studies revealing the ontogeny of distribution and gene expression of these neuropeptides in the brain of different groups of animals. Due to restrictions regarding the length of this paper, the interesting issue of the distribution and gene expression of neuropeptides during brain development in models of rodents and pigs were emphasised. It was also necessary to reduce the number of neuropeptides discussed to those receiving the most attention by researchers in recent years. 


\section{Luteinising hormone-releasing hormone (Figures 1-5)}

One of the neuropeptides belonging to the hypothalamic releasing and inhibiting hormone family is luteinising hormone-releasing hormone (LHRH). LHRH is a decapeptide that regulates reproductive function and behaviours in mammalian species. Because of the importance of efficient reproductive function in farm animals, the ontogeny of LHRH immunoreactivity (LHRH-IR) was also studied in the pig (Polkowska et al., 1985; Danchin and Dubois 1982). $\mathrm{LHRH}$ was present in nerve terminals of the median eminence (ME) and organum vasculosum of the lamina terminalis (OVLT) beginning on day 72 of gestation (G72) and gradually increased with development of the embryo. In this stage, a few immunoreactive LHRH perikarya appeared, but they were most numerous in the last days of pregnancy (G110; duration of pregnancy averages 114 days). The greatest numbers of these neurons were localised in the most anterior periventricular parts of the hypothalamus, the preoptic area (POA) and diagonal band of Broca, but they were very rare in the medial basal hypothalamus $(\mathrm{MBH})$.

The ontogeny of LHRH-IR was studied in the Meishan pig by Pearson et al. (1996b). LHRHIR cell bodies and fibres were detected entering the brain via the terminal nerve and in the septal region of the basal telencephalon at G30. The number of immunoreactive cell bodies increased at G50 and cells were localised primarily in the septum, OVLT, POA and lateral hypothalamus, whereas immunoreactive fibres were present throughout the septum and hypothalamus and reached the ME. The density and distribution of immunoreactive fibres increased by G70 and G90, but did not change dramatically from G90 to postnatal day 50 (P50). These results indicate that LHRH may be present earlier in the Meishan pig brain than in the domestic pig. Nerve fibres containing LHRH-IR appear to reach the ME in the Meishan pig 10 days earlier than previously reported for the domestic pig. Some quantitative analyses of LHRH content in the porcine brain were performed. The POA and suprachiasmatic area contained only low concentrations of $\mathrm{LHRH}$ while the $\mathrm{MBH}$ contained high concentrations of $\mathrm{LHRH}$ (Kumar et al., 1991).

\section{VIP-glucagon family peptides (Figures 6-8)}

The VIP-glucagon family of peptides encompasses, among others, glucagon-like peptide-1, peptide histidine isoleucine, pituitary adenylate cyclase-activating polypeptide (PACAP), and vasoactive intestinal peptide (VIP). VIP is a 28-amino acid neuropeptide, which has potent growth-related actions on dissociated neural cells (Gressens 1998, Waschek 1995). In recent years, its role in brain development has been elucidated: VIP is a regulator of early neurodevelopment and embryonic growth, a stimulator of neocortical astrocytogenesis, and is a neuroprotective molecule acting against excitotoxic and other neurotoxic substances.

Localization of VIP-immunoreactive (VIP-IR) structures and appearance during development were studied in rats (Hajos et al., 1990). Numerous cells displayed intense VIP-IR in the subventricular zone of the rat occipital hemisphere from birth to P8 (Hajos et al., 1990). By P8, this cell population was markedly reduced. In the ageing brain (Cha et al., 1995), there was a significant loss of VIP-IR neurons in the cortex. Numbers of VIP-IR structures increased in the developing visual cortex of the rat (Zilles et al., 1991). In studies by Ichihara et al. (1983), VIP concentrations increased in some brain areas until the fourth week of postnatal life, and then decreased in brains of adult animals. Similar observations regarding the suprachiasmatic nucleus (SCN) were described by Chee et al. (1988), who revealed a $36 \%$ decrease in the number of VIP-IR neurons in the SCN of old rats compared to that of young animals. Concentrations of VIP were determined in extracts of the brain of the developing and adult pig (Ichihara et al., 1984). VIP concentrations in acid extracts of the brain cortex were $13.5+/-2 \mathrm{pmol} / \mathrm{g}$ at birth and rose 

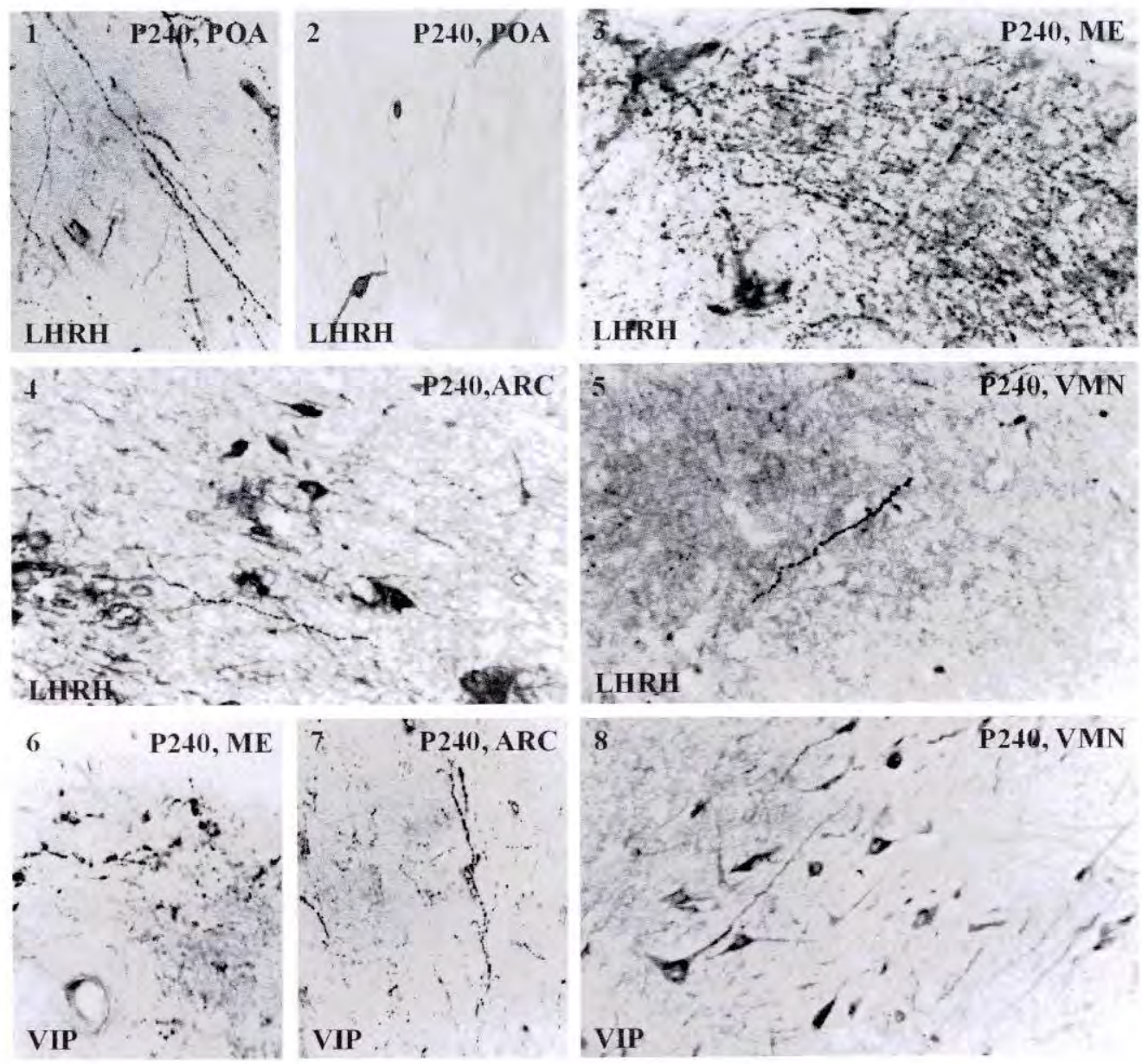

Figures 1-8. Immunocytochemical localization of LHRH in the median eminence (ME), preoptic area (POA), arcuate nucleus (ARC) and venteromedial nucleus (VMN) and VIP in the ME, ARC and $\mathrm{VMN}$ in a sexually mature pig.

1. LHRH-IR nerve fibres in the POA.

2. LHRH-IR nerve cell bodies in the POA.

3. Very dense meshwork of LHRH-IR nerve fibres in the ME.

4. LHRH-IR neurons and nerve terminals in the ARC.

5. LHRH-IR nerve fibres in theVMN.

6. VIP-IR nerve fibres in the ME.

7. VIP-IR nerve fibres in the ARC.

8. Numerous VIP-IR neurons in the VMN.

gradually to $30+/-9 \mathrm{pmol} / \mathrm{g}$ in the adult. Localization of VIP-IR structures was studied in the hypothalamus of the immature female pig (Calka 1992). VIP-IR neurons were found in the arcuate (ARC), paraventricular (PVN) and supraoptic nuclei (SON), as well as in the pituitary stalk. The ME contained an abundance of VIP-IR processes, both in the internal and external layers. In the developing porcine hypothalamus, VIP-IR nerve fibres and perikarya were observed in the POA, SON, PVN, ME, ARC, ventromedial nucleus $(\mathrm{VMN})$ and mamillary region (W. Sienkiewicz, unpublished). Animals at P1 and sexually mature pigs were used in this 
study. Some nerve fibres and cell bodies were detected in the POA of pigs at P1. There were single nerve fibres in the SON, while perikarya were moderate in number. The PVN contained a moderate number of neuronal somata and numerous nerve fibres, which were gathered mainly in the periventricular zone. During maturation, there were no changes in number of VIP-IR structures in these nuclei. A greater number of nerve fibres were in the ME (Fig. 6), ARC (Fig. 7) and VMN (Fig. 8) in sexually mature pigs compared to 1 day-old animals. Neuronal somata were absent at P1, but were numerous in the adults (Fig. 8 ). There were single VIP-IR fibres and neurons in nuclei of the mamillary region in both P1 and adult pigs (W. Sienkiewicz, unpublished observations).

In studies by Waschek et al. (1996), the VIP gene was expressed at least as early as G11 in the mouse hindbrain. VIP expression was also studied during rat brain development (Graber and Burgunder 1996). VIP was first expressed in the lateral thalamus at G17. At G19, VIP mRNA was also in the hypothalamus, especially the SCN. VIP expression matured continuously during the first three postnatal weeks, and adult-like patterns were found at P22, when expression of VIP was most prominent in the cerebral cortex, thalamic nuclei and SCN. Gozes et al. (1988) described high VIP mRNA expression as early as G16, but VIP mRNA expression was significantly lower in the cerebral cortex in aging rats compared to P21 rats. The VIP mRNA, VIP protein and VIP receptor-associated protein were in endothelial cells of veins, arteries and capillaries in the marginal zone of brainstem tissue sections, especially in the pons and mesencephalon, as well as in pial vessels of newborn pigs (Lange et al., 1999).

\section{Neuropeptide Y (Figures 9 and 10)}

Neuropeptide Y (NPY) and related peptides (pancreatic polypeptide and peptide tyrosine-tyrosine) are some of the most intensively studied neuropeptides. Localization and development of NPY-containing structures within the brain was studied in a wide range of species, including farm animals.

Changes in the concentration of NPY were determined in the developing rat brain (Allen et al., 1984). NPY was present in the brain stem and diencephalon in the earliest embryos studied (G14). Postnatally, concentrations of NPY rose rapidly in all regions examined. The distribution of NPY in the developing rat brain was also studied by immunocytochemistry (Woodhams et al., 1985). NPY-IR perikarya first appeared on G13 and immunostained fibres appeared from G15 onwards: staining of the perikaryal was generally more intense prenatally than after birth. According to other authors (Kagotani et al., 1989), NPY-IR neurons first appeared in the rat ARC and in the dorsolateral hypothalamic area on G14.5. NPY-IR cell bodies increased in number in the ARC, dorsomedial-lateral hypothalamic area and PVN until birth, but disappeared thereafter, with some cells still remaining in the ARC. On the contrary, the number of NPY-IR fibres diminished markedly on P16, particularly in the PVN, dorsomedial-lateral nuclei, and medial $\mathrm{POA}$, but recovered considerably on $\mathrm{P} 60$ and P120.

During the ontogeny of the porcine brain, NPY-IR in cell bodies and fibres was evident in many areas of the brain at G30, including the basal telencephalon, hypothalamus, mesencephaIon, pons and medulla (Pearson et al., 1996c). Throughout prenatal development, cell bodies containing NPY-IR generally increased in number and distribution in the brain (Fig. 9, 10). The distribution of NPY-IR in fibres became more widespread as gestation progressed, showing a pattern by G110 that was characteristic of the postnatal period. The intensity of NPY-IR in fibres also increased throughout gestation. During postnatal development, the number of cell bodies displaying NPY-IR decreased. There was a dramatic reduction in the number of immunoreactive cell bodies in the ARC between P1 and just before weaning at P20. Some additional 

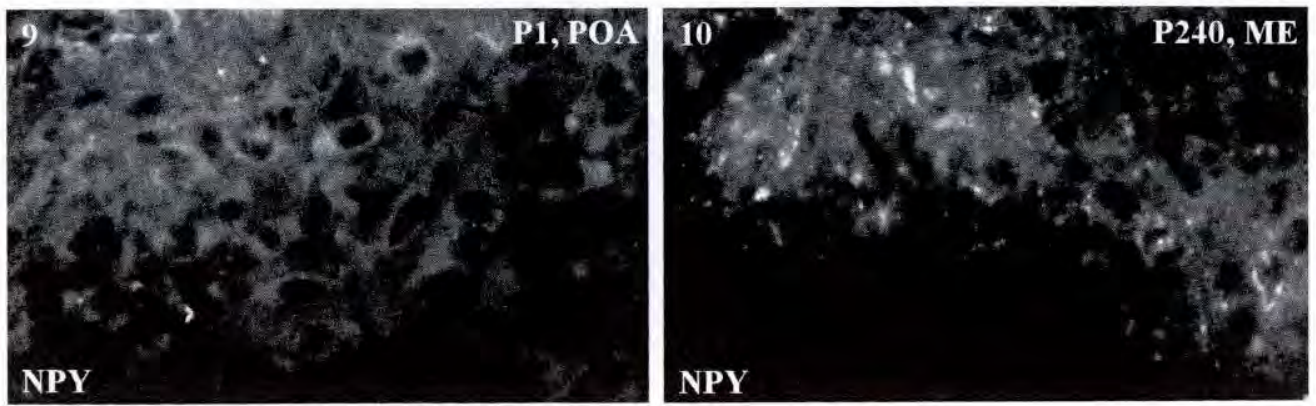

Figures 9 and 10. Immunocytochemical localization of NPY in the preoptic area (POA) of a 1 dayold pig and ME of a sexually mature pig.

9. NPY-IR nerve cell bodies in the preoptic area in a 1 day-old pig.

10. NPY-IR nerve fibres in the median eminence in a sexually mature pig.

increases in immunoreactivity occurred postnatally, especially in the periventricular hypothalamus and the hippocampus. NPY-IR decreased postnatally in other brain areas, like the caudate nucleus and putamen. In juvenile pigs, NPY-IR hypothalamic structures were present in the POA, ARC, dorsomedial nucleus (DMN) and VMN, as well as in the internal layer of the ME and infundibulum (Calka et al., 1994). There were NPY-IR fibres throughout the hypothalamus, but most prominently within the periventricular region.

Expression of NPY at the mRNA level was studied in the rat brain. In the normal adult rat, hypothalamic NPY mRNA expression is limited to the ARC. In contrast to the normal adult rat, there was NPY mRNA in the ARC, the DMN and the perifornical region during development (Singer et al., 2000). NPY mRNA expression in all three regions increased progressively from $P$ 0-4 reaching maximum at P16 and subsequently decreasing to near-adult expression by P30. Similar observations were described by Grove et al. (2001). Examination of the full rostral to caudal extent of the hypothalamus revealed two additional regions displaying novel NPY mRNA expression; the parvocellular division of the PVN and lateral hypothalamus.

\section{Galanin (Fig. 11-16)}

Development of galanin (GAL)-IR in the rat CNS has been investigated immunologically (Sizer et al., 1990). GAL-IR processes in the CNS were first recognised in the spinal cord at P1. The GAL-IR neuronal somata were first visualised in several regions of the diencephalon at P2. Positive cells were also detected in many brainstem areas at later stages of maturation. The number, density and staining intensity of GAL-IR structures in these and other brain regions increased steadily until P28, at which age the adult distribution was attained. No reduction in GAL-IR was noted in any area during later postnatal periods. GAL-IR cell bodies and fibres were evident primarily in the porcine hypothalamus at G30 (Pearson et al., 1996a)., Cell bodies containing GAL-IR generally increased in number and distribution in the brain throughout the prenatal period. During postnatal development, the number of GAL-IR cell bodies decreased, particularly in hypothalamic areas (Fig. 11-16). The presence of GAL-IR fibres became more widespread throughout gestational development, establishing a pattern by postnatal P1 that continued during later postnatal ages. The intensity of GAL-IR in fibres also increased throughout gestation. Some additional increases in immunoreactivity occurred postnatally, especially in the periventricular hypothalamus. 

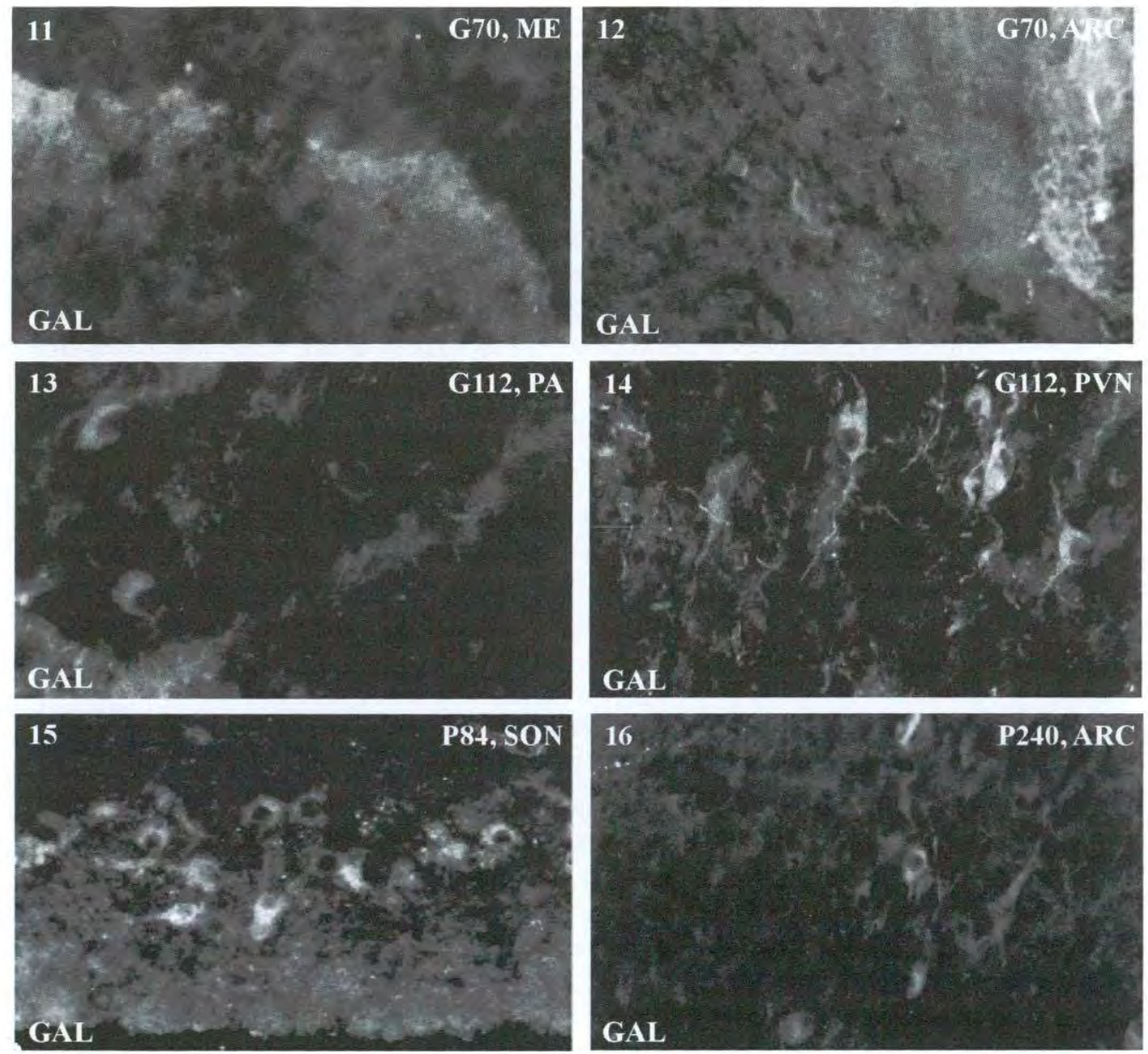

Figures 11-16. Immunocytochemical localization of GAL in the hypothalamus of the pig.

11. Gal-IR nerve fibres in the ME of a 70 day-old foetus.

12. Gal-IR nerve cell bodies in the ARC of a 70 day-old foetus.

13. Gal-IR perikarya in the POA of a 112 day-old foetus.

14. Gal-IR perikarya in the PVN of a 112 day-old foetus.

15. Gal-IR neurons in the SON of an 84 day-old pig.

16. Gal nerve cell bodies in the ARC of a sexually mature pig.

The regional and cellular distribution of GAL-like peptide (GALP) mRNA (Larm and Gundlach 2000), GAL peptide and GAL receptor-binding mRNAs (Gundlach et al., 2001) and preprogalanin messenger RNA (Ryan and Gundlach 1996) were investigated in the rat brain. In a thorough screening of the adult male rat brain, GALP mRNA expression was detected only throughout the rostrocaudal extent of the ARC with the strongest hybridisation signal in the posterior and periventricular zones (Larm and Gundlach 2000). GALP mRNA-positive neurons were mostly localised in the ventromedial division of the ARC. Smaller numbers of labelled neurons were also in ventrolateral areas. The distribution of GALP mRNA was somewhat complementary to that of GAL mRNA in the ARC, but contrasted with the broad distribution of this transcript 
throughout the hypothalamus. GAL mRNA was also distributed along the rostrocaudal extent of the ARC, but was most abundant at the anterior to middle levels and in ventrolateral regions. In the adult rat brain, GALP mRNA expression was restricted to a discrete subpopulation of neurons in the ARC. Expression and distribution of the mRNA coding for the GAL precursor, preprogalanin (ppGAL), were also analysed in the rat periventricular nuclei, PVN, SON, DMN, ARC nuclei and the lateral hypothalamic area (Giorgi et al., 1995). During development, ppGAL mRNA was detected in all the nuclei considered beginning at P1. Preprogalanin mRNA progressively increased from P8 to P21. The ontogenic expression of ppGAL mRNA in the postnatal rat brain was studied by Ryan et al. (1997). Preprogalanin mRNA was detected in the forebrain and hindbrain from P4 to P60 (adult). Weak hybridisation signals were observed in cells of a number of nuclei, including the medial POA, PVN and DMN on P4.

\section{Tachykinins}

Four neuropeptides belong to the tachykinin family: neurokinin A, neurokinin B, neuropeptide $K$ and Substance $P(S P)$. Most data regarding development of the tachykinin brain system were obtained from studies of SP-containing structures.

Ontogeny of the SP neuron system in the rat brain stem was investigated by Sakanaka et al. (1982). SP-IR structures (cells and fibres) first appeared in the primordium of the ventral part of the nucleus tractus spinalis nervi trigemini $(n V s)$, in the reticular formation between nucleus reticularis lateralis and $\mathrm{nVs}$, and in the reticular formation between $\mathrm{nVs}$ and nucleus originis nervi facialis at G14 in the brain stem. After that time, SP-IR structures made their appearance gradually in various areas of the brain stem. Ontogeny of SP-IR structures was also examined in the VMN of the rat (Yamano et al., 1984). SP-IR cell bodies and fibres first appeared at G21. These structures subsequently increased in number. The maximum content was reached at P21 and was observed even in adult rats.

The concentrations of other members of the tachykinin family: neurokinin A, neurokinin B and neuropeptide $\mathrm{K}$, were measured in several regions of the rat CNS at different stages of postnatal development by Diez-Guerra et al. (1989). All 3 tachykinins were detectable in the hypothalamus, striatum, substantia nigra, cerebral cortex and spinal cord during postnatal development. Interestingly, there was a general increase in tachykinin concentrations during the second week of life. Some of these reached concentrations on P15, which far exceeded those in the adult. Tachykinin content of most areas declined slowly after P15 until adult values were finally reached. There is no information on development of tachykinin-containing structures in the brain of large domestic animals. The distribution of SP in the brain and spinal cord of the adult pig was described for the first time in a paper by Kumar et al. (1991). There were high levels of SP in the POA, suprachiasmatic area, MBH and brain stem, while moderate amounts of SP were in the olfactory bulb. In our studies, the number of nerve cell bodies in the POA and nerve fibres in the SON decreased, whereas the number of nerve fibres remained unchanged in the PVN, ME and mamillary region when comparing sexually mature pigs to animals at P1. The number of nerve fibres increased in the POA, whereas the number of neuronal somata increased in the SON and ARC (W. Sienkiewicz, unpublished). Postnatal development of SP-IR structures was studied in the pineal gland of newborn, P21 and 7 month-old female pigs (Przybylska-Gornowicz et al., 2000). Immunoreactive nerve fibres were in the pineal gland as well as in the posterior commissure and habenular areas and SP-IR cell bodies were in the medial habenular nucleus, but there were no differences in the distribution and density of SPIR nerve fibres among newborn, P21 and 7 month-old pigs. SP-containing structures in the MEARC complex in the juvenile pig were also described by Sienkiewicz et al. (1996). Numerous 
SP-IR perivascular nerve fibres were located within both layers of the ME. SP-IR perikarya were also in the ARC.

Gene expression of tachykinins was studied in the developing and ageing rat brain. Preprotachykinin A (PPT-A) mRNA-positive and -immunoreactive cells were first detected at birth. By P5, the numbers of SP-expressing cells diminished dramatically. The number of SP mRNApositive neurons and SP-IR cells decreased gradually from P10 and P15 onward (Zhang and Harlan 1994). In contrast, expression of neurokinin B was not evident until P10. Neurons expressing this tachykinin were gathered in layer II, and to a lesser extent in layers $\mathrm{V}$ and VI of the cerebrum. This distribution pattern remained at P45 (Zhang and Harlan 1994). In studies by Brene et al. (1990), rat brains at 12 different developmental stages, ranging from G15 to adult were used. Preprotachykinin A mRNA was detected as early as G15 and an approximately 3-fold increase occurred by birth, which remained until P21 when the level increased, reaching a peak at P35. Adult concentrations were approximately 3-fold higher than levels at birth. The distribution of PPT-A mRNA-expressing cells was studied on sections of G20, P4 and P7 rat brains as well as from adults (Brene et al., 1990). Cells expressing PPT-A mRNA were detected in the forebrain at all 4 stages analysed. However, the hybridisation pattern and labelling intensity varied in different brain regions during development. In the cingulate cortex, there was intense PPT-A labelling in numerous cells at G20 and P4 and 7 , whereas only a few scattered labelled cells were in the adult cingulate cortex. Labelled cells were found in the frontoparietal cortex from P4 to adult, with the highest density of labelled cells at P7. There were also developmental differences in both the distribution of PPT-A mRNAexpressing cells and the level of PPT-A mRNA expression in the caudate-putamen, lateral hypothalamus and amygdala.

The effect of ageing on PPT-A mRNA levels in discrete regions of the rat brain was studied by Pompei et al. (1999). There were no changes in PPT-A mRNA levels in the nucleus accumbens, islands of Calleja, and medial POA.

\section{Opioid peptides}

The opioid peptide family, which is derived from proopiomelanocortin (POMC), consists of enkephalins (ENK), endorphins (END), dynorphins (DYN) and their other forms. Development of leucine-ENK and DYN-A was studied in the rat CNS and pituitary gland (Khachaturian et al., 1983). POMC-IR appeared first in hypothalamic neurons on G12, in pituitary anterior lobe cells on G15, in pituitary intermediate lobe cells on G16, and in perikarya of the nucleus tractus solitarius on G17. At the foetal stages, G19-22, all POMC systems were adult-like; however, the peak of IR occurred between P21 and P28. In contrast, both Leu-ENK-IR and DYN-A-IR appeared at later embryonic stages, G16-17; their maturation lagged behind that of POMC peptides and the peak of IR for these latter peptides also occurred between P21 and P28. The effect of ageing on B-END in rat corpus striatum and hypothalamus was investigated in the studies of Gambert et al. (1980). In both brain areas, basal levels of ß-END declined with age. B-END-IR was quantitated in the $\mathrm{MBH}$ in rats of different ages (P1-P45) by radioimmunoassay (Hompes et al., 1982). Low, but significant, amounts of $ß-E N D$ were present on P1. Hypothalamic ß-END content did not change during the first week, but decreased during the second week to a minimum on P14. Thereafter, B-END-IR increased rapidly to P28 and remained at this level. Also, Tsang and co-workers (1982) studied postnatal changes in levels of ENK and B-END in rat cerebellum, brainstem and whole forebrain. The opioid peptides reached their highest levels at the first week postpartum in the cerebellum, at the second week in the brainstem and at the third week in the whole forebrain, indicating a caudalrostral sequence of development. Immunoreactive ß-END was measured in discrete brain areas and pituitary glands from rats aged 6 weeks (young), 6 months (mature), and 20-24 months (senescent) (Gambert et al., 1980). B-END declined significantly with age in the hypothalamus and 
corpus striatum. B-END increased in the frontal lobe during early development; however, no change was noted after maturity. The distribution of END-IR was described in the dentate area, hippocampus, and subiculum of the pig by Holm et al. (1993). ENK-IR nerve cell bodies were faintly stained and generally present in very small numbers, except for some pyramidal cells in the subicular cell layer. ENK-like immunoreactive fibres were few in number, whereas stained puncta appeared with variable densities. There were puncta of particularly high densities in the dentate molecular layer, whereas they were of moderate density in the dentate hilus, stratum moleculare of the hippocampal regio superior, and in the subiculum. In the porcine hypothalamus at P1, some Leu5-Enk-IR nerve fibres were found in the POA, SON, PVN, ME, ARC and mamillary region. The ARC of immature female pigs contained a moderate number of nerve cell bodies and also a moderate number of nerve fibres were in the ARC and ME (Sienkiewicz et al., 1996). In sexually mature pigs, the POA exhibited a moderate number of nerve fibres (Fig.17) and cell bodies. The SON contained a moderate number of perikarya and single nerve fibres. Single nerve cells and numerous nerve fibres were in the PVN. The ME had a very dense network of nerve fibres (Fig. 18) and numerous neurons were visible in the ARC (Fig. 19) and VMN (Fig. 20). The mamillary region exhibited single nerve fibres and cell bodies (W. Sienkiewicz, unpublished).

Levels of proenkephalin A (P-enk A) and B mRNA were analysed throughout prenatal development in the hippocampus and striatum of foetal pigs by Pittius et al. (1987a). In the hippocampus, both mRNAs increased steadily throughout development with P-enk B mRNA increasing faster than P-enk A mRNA. In the striatum, both mRNAs behaved similarly, increasing to a maximum level around mid-gestation and declining thereafter.

Concentrations of P-enk B mRNA in porcine brain and pituitary were also measured using RNA blotting and solution hybridisation (Pittius et al., 1987b). P-enk B mRNA concentrations were highest in the caudate nucleus, followed by hypothalamus and hippocampus.

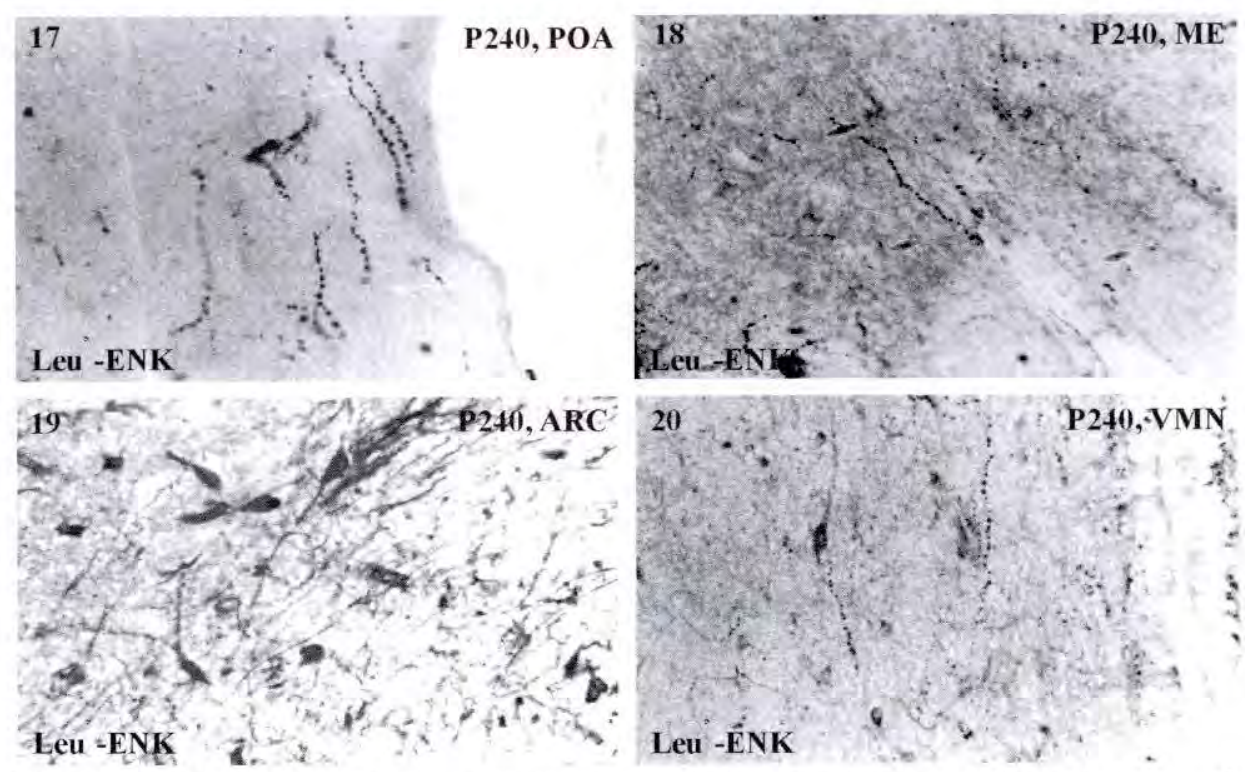

Figures 17-20. Immunocytochemical localization of Leu' ${ }^{5}-E N K-I R$ in the hypothalamus of a sexually mature pig.

17. Leu 5 -ENK-IR nerve fibres in the POA.

18. Leu $^{5}$-ENK-IR nerve fibres in the ME.

19. Leu ${ }^{5}$-ENK-IR nerve cell bodies in the ARC.

20. Leu ${ }^{5}-E N K-I R$ ner ve cell bodies and nerve fibres in the VMN. 


\section{Catecholamines (Figs. 21-28)}

A very important group of neurotransmitters, which strongly influence different brain functions are the catecholamines. Development of the catecholaminergic system was intensively studied in the porcine brain, especially with respect to the hypothalamus, by Sienkiewicz et al. (1996), Sienkiewicz (2002) and Sienkiewicz and Lakomy (2003). In the porcine POA at G70, there were single nerve fibres immunoreactive only for tyrosine hydroxylase (TH) (Calka et al., 1993b; Fig. 21). These nerve fibres were encountered in all three nuclei of the POA. The most numerous TH nerve fibres were found in the medial preoptic nucleus and only a very few fibres were observed in median preoptic nucleus. There were additional single nerve cell bodies immunoreactive to $\mathrm{TH}$ in the POA at G84, and some of them also contained dopamine betahydroxylase $(\mathrm{D} B H)$. These nerve fibres were slightly more numerous at G112 (Fig. 22). Moderate numbers of nerve fibres immunoreactive to $\mathrm{TH}$ and only single TH/DßH-IR nerve terminals were in the POA at P1. Furthermore, TH-IR nerve cell bodies were moderate in number and many of them also contained DBH-IR. A moderate number of nerve fibres were in the POA of 10 week-old pigs. They contained mainly TH-IR, but part of them were also TH/DßH-IR. Very few nerve fibres, which contained only DBH-IR, were present. Moderate numbers of THIR nerve cell bodies were in this area, and some of them also contained DßH-IR. Only perikarya containing phenylethanolamine $\mathrm{N}$-methyltransferase (PNMT)-IR, but not TH were encountered. TH-IR nerve cell bodies (Fig. 23) and fibres (Fig. 23) were numerous in the POA of sexually mature sows. Part of them simultaneously contained DßH-IR. Single perikarya also stained for PNMT.

There were single TH-IR neurons in the ARC of G70 foetuses and single TH-IR neurons as well as single fine TH-IR nerve fibres in G84 foetuses. The number of TH-IR nerve fibres increased in foetuses by G112. There was a small increase in the number of TH-IR nerve cells bodies, but a significant decrease in the number of TH-IR nerve fibres in P1 animals. Numerous nerve cells bodies and nerve fibres, which were TH-, DBH-, TH/DBH- and PNMT-IR, were in the ARC of 10 week-old piglets. Moreover, TH-IR fibres were most numerous, whereas PNMTIR nerve terminals were only occasionally encountered. In sexually mature sows, the immunoreactive structures resembled those in 10 week-old pigs.

Single nerve fibres, which were immunoreactive only for $\mathrm{TH}$, were in the ME of G70 foetuses. The number of TH-IR nerve fibres was higher in the ME of G84 and G112 foetuses as compared to G70 foetuses. The ME contained numerous TH-IR and single DßH-IR nerve fibres in P1 animals. There were greater numbers of TH- and DBH-IR nerve terminals in the ME of 10 week-old and sexually mature animals as compared to younger animals. The density of these nerve fibres increased with advancing age. TH-IR nerve terminals appeared in the ME before P70, whereas DßH-IR nerve terminals appeared for the first time at P1.

Other hypothalamic nuclei were also studied. In general, the number of catecholamine synthesizing enzyme-IR structures also increased with age in the AHA, SON, VMN (Fig. 2528) and tubero-mamillar (TM) nuclei. However, in the PVN, the number of IR structures increased up to P70, and then decreased in sexually mature pigs to half of the amount observed earlier.

\section{Interaction between developing LHRH and peptidergic systems}

All the substances mentioned above influence $\mathrm{LH}$ release. VIP inhibits secretion of LH by a hypothalamic action (Weick and Stobie 1992; Weick and Stobie 1995; Van der Beek et al., 1999). Experiments involving neural lesions indicated that there are VIP receptors on neurons in the PVN whose cell bodies are located in the SCN. VIP also blocked the steroid-induced LH 

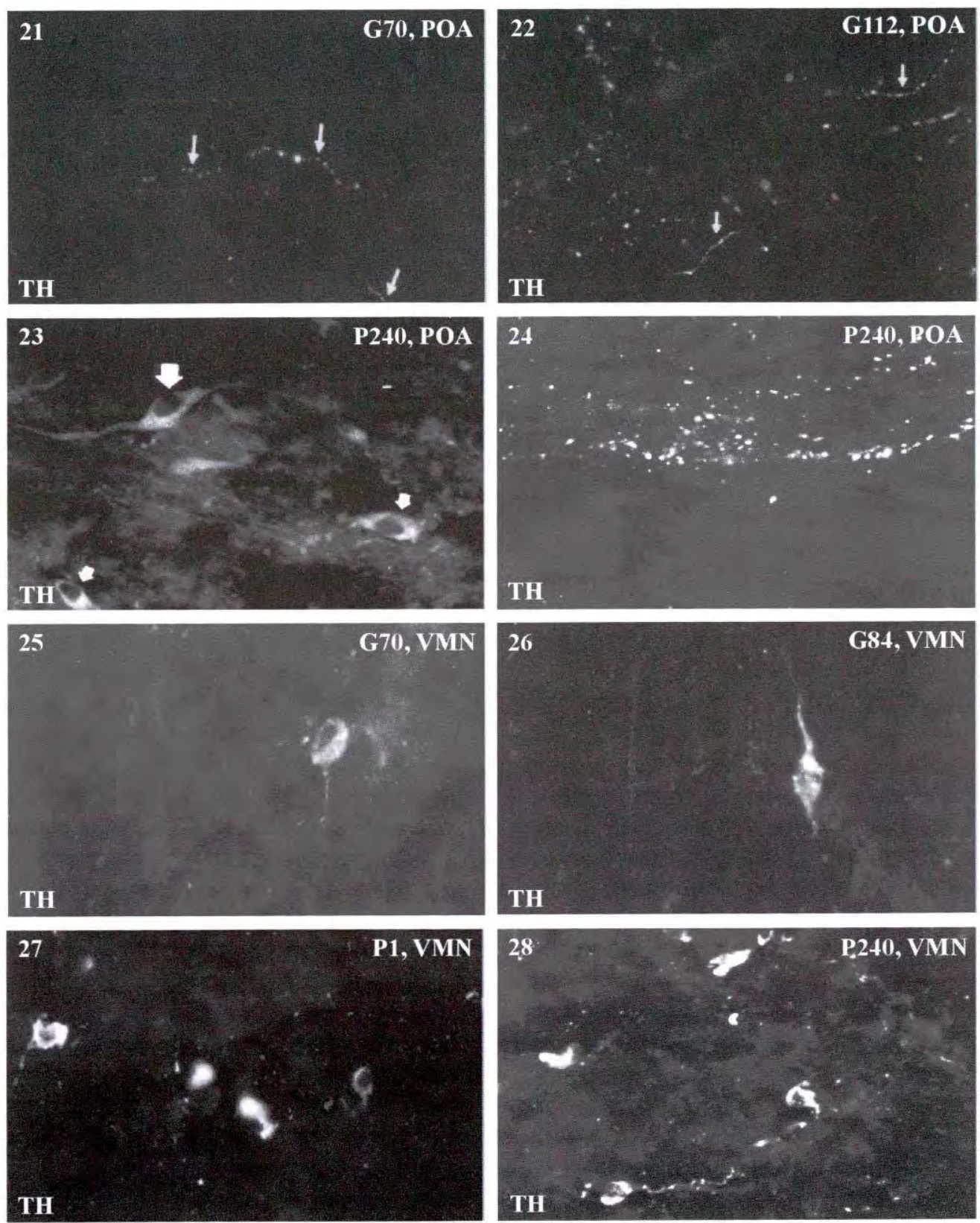

Figures 21-28. Immunocytochemical localizationof tyrosine hydroxylase (TH) in the hypothalamus of a sexually mature pig.

21. TH-IR nerve fibres in the POA in a 70 day-old porcine foetus.

22. TH-IR nerve fibres in the POA in a 112 day-old porcine foetus.

23. TH-IR perikarya in the POA in a sexually mature pig.

24. TH-IR nerve fibres in the POA in a sexually mature pig.

25. TH-IR neuron in the VMN in a 70 day-old porcine foetus.

26. TH-IR neuron in the VMN in a 84 day-old porcine foetus.

27. TH-IR neurons in the VMN in a 1 day-old piglet.

28. TH-IR neurons in the VMN in a sexually mature pig. 
surge in the ovariectomised rat (Weick and Stobie 1992). A VIP antagonist induced a small, but significant increase in LH secretion in the intact rat, indicating that VIP has a modulatory rather than a regulatory role in control of $\mathrm{LH}$ secretion. Also, about $40 \%$ of all $\mathrm{LHRH}$ neurons analysed contained VIP2 receptors. In addition processes, which contained VIP, were in close apposition to a significant number of VIP2 receptor-positive LHRH neurons (Smith et al., 2000). LHRH neurons, that exhibited immunoreactivity for the VIP2 receptor, are located predominantly in the OVLT. In the ME, where the majority of LHRH neurons terminate, VIP2 receptor IR was absent. These findings showed that VIP neurons may communicate directly with LHRH neurons (Smith et al., 2000). The data presented above demonstrate the importance of the VIP-IR system for LH release. It is very interesting to compare time of appearance of VIP-IR and LHRH-IR structures within the porcine brain, especially in the hypothalamus. Unfortunately, data regarding development of hypothalamic VIP-IR structures in the pig are fragmentary, but generally indicate that the number of VIP-positive neuronal elements in the hypothalamus increase with age up to the prepubertal period. The most numerous VIP-IR nerve structures were in juvenile gilts, and there was a decrease in the number of those nerve structures in sexually mature pigs. Similarly, the number of LHRH-IR structures increased with age, up to the end of foetal development. Later, there were no changes in the number of LHRH-IR structures until the peripubertal period when LHRH-IR increased significantly. There was a similar pattern of changes in hypothalamic LHRH mRNA. These similarities in development of the VIP and LHRH hypothalamic systems suggest that VIP is important for development and survival of LHRH containing neurons. Direct contact between VIP-IR and LHRH-IR structures is additional evidence for the importance of VIP in regulation of $\mathrm{LH}$ secretion via the hypothalamic LHRH system.

NPY also modulates LH secretion by influencing the LHRH hypothalamic system (McDonald 1990; Calka et al., 1993a; Crowley and Kalra 1994; Advis et al., 2003) and by acting directly on LH producing cells in the pituitary (McDonald 1990; Bauer-Dantoin et al., 1991; Kalra and Crowley 1992; Estrada et al., 2003). NPY-IR appeared in porcine hypothalamic centres earlier than LHRH-IR structures, but later in the course of development, the localization patterns of these substances were similar. The number of NPY-IR neurons and nerve terminals as well as LHRH mRNA declined during the peripubertal period. In sexually mature animals, the number of NPY-IR neurons increased in hypothalamic centres, especially in the PVN. At the time of the preovulatory LH surge, NPY gene expression increased in the ARC of the rat. These results suggest that interactions between NPY and LHRH neurons are important in controlling the timing, magnitude and maintenance of the preovulatory $\mathrm{LH}$ surge.

Results of previous studies clearly show that GAL is also a very important agent in regulation of $\mathrm{LH}$ release due to excitatory effects on $\mathrm{LHRH}$, and therefore, $\mathrm{LH}$ release (Lopez and NegroVilar 1990; Lopez et al., 1993; Elsaesser 2001). GAL-IR structures were first visible in hypothalamic areas on G30. The number of GAL-IR structures reached maximum development at P1, then gradually decreased, except in the periventricular hypothalamus where GAL-IR structures increased. In adult female mice, many LHRH-containing neurons also showed IR for GAL (Rajendren and Gibson 1999). Galanin mRNA expression may be regulated by sex steroids, since it was induced coincident with the $\mathrm{LH}$ surge, and persisted well after completion of the LH surge (Finn et al., 1998). LHRH-containing cells begin to express detectable levels of GAL mRNA at the onset of puberty. During the oestrous cycle, GAL mRNA levels increased in $\mathrm{LHRH}$ neurons at the time of the LH surge (Rossmanith et al., 1996). Similarities between patterns of development of GAL and LHRH systems and the data reviewed above, suggest a tight coupling of GAL biosynthesis and release to the activity of the LHRH neurons.

Tachykinins, which include SP, suppress LH release (Battmann et al., 1991; Sahu and Kalra 1992; Duval et al., 1996; Hidalgo-Diaz et al., 1998). The previously mentioned papers clearly 
demonstrated that SP plays a modulatory role in the neuroendocrine control of the preovulatory L.H surge. There are some differences between the development of the SP-IR and LHRH-IR hypothalamic systems. The number of SP-IR perikarya was lower, but the number of SP-iR nerve fibres was greater in the POA and SON of adult female pigs compared to $\mathrm{P} 1$, whereas the number of nerve fibres in the PVN, ME and mamillary region remained unchanged between $\mathrm{P} 1$ and sexual maturity. A greater number of SP-IR neuronal somata was in the ARC of the sexually mature pigs. The lower number of SP-IR perikarya and the greater number of SP-IR nerve fibres in the adult pig may have resulted from release of $S P$ from the neurons.

Opioid peptides suppress LH release (Barb et al., 1986; Rossmanith et al., 1988; Barb et al., 1994; Giri and Kaufman 1994; Lieberman et al., 1998). Studies in rodents and pigs also suggest a role for the opioid peptide system in controlling the timing of the LH surge (Asanovich et al., 1998, Barb et al., 1986, Barb et al 1994). Based on this information, it is interesting to compare time of appearance of opioid peptides and LHRH-IR in the porcine hypothalamus. During postnatal development, there is a gradual increase in Leu5-ENK-IR structures, which reach a maximum in sexually mature pigs, so it can be assumed that this system develops simultaneously with the LHRH system in the hypothalamus.

Catecholamines are one of the most powerful stimulators of LHRH and LH release from the hypothalamus and pituitary, respectively (Sarkar and Fink 1981; Barraclough 1983; Kalra and Gallo 1983; Kalra 1985; Bergen and Leung 1986; Clement et al., 1986). Catecholaminergic cells are also sensitive to changes in the hormonal milieu during the oestrous cycle (Conde et al., 1995) and are most active during early proestrus. Taking into consideration all these data, it is interesting to compare time courses of development of catecholamine and LHRH structures in the pig hypothalamus. The catecholamine system develops gradually with age, reaching its final configuration in sexually mature animals, especially in regions involved in regulation of sexual function, such as the POA, ARC, ME and MBH. This similar pattern of development compared to the LHRH system indicates functional relationships between these systems. In summary, comparison of development of neurotransmitter structures with development of the LHRH-IR system within hypothalamus revealed similarities and differences in the time course of these processes. The hypothalamic catecholaminergic system, which stimulates LHRH and LH release, develops in a very similar manner to the LHRH-IR system. The number of catecholamine positive LHRH-IR structures gradually increases throughout development and reach a maximum in the sexually mature pig. NPY-IR structures are already developed in the hypothalamus at the end of foetal life, but later, at the beginning of postnatal life, the number of these structures decreases, which is earlier than when LHRH declines. However, NPY-IR structures increase in later periods. The number of GAL-IR structures in the hypothalamus reach maximum development at $\mathrm{P} 1$, then gradually decrease, except in the periventricular hypothalamus where GAL-IR structures increased. VIP-IR structures were studied in the postnatal porcine hypothalamus. Available data indicate that the VIP-IR system is already well developed in P1 piglets and there are no changes when compared to adults, except in the ME and ARC nucleus where an increased number of VIP-IR structures are found in adults. Hypothalamic systems containing SP and Leu ${ }^{5}$ ENK develop gradually in the pig, reaching their final configuration in adults. The different patterns of development of the hypothalamic systems containing substances discussed above indicates different roles by which they influence maturation of LHRH-IR structures and LHRH and LH release. The decrease in the number of structures containing VIP and GAL, coincident with the time when the LHRH system almost reaches its final configuration, supports the idea that those substances are very important agents for survival, growth and maturation of LHRH neurons. Structures which are IR for other substances also increased during development. Catecholamines and NPY excite, whereas SP and opiates inhibit LHRH and LH release. To completely understand all aspects of the regulation of $\mathrm{LHRH}$ synthesis and release from hypothalamic centres, further studies are needed. 
All of the above mentioned substances, which influence the LHRH system, are present in hypothalamic centres involved in regulation of reproductive functions of sexually mature animals, so it is extremely difficult to explain how they interact to regulate such a complicated and sensitive process. The results presented above strongly indicate that timing of the appearance and changes in the quantity of these substances are crucial for proper regulation of reproductive function.

\section{Reference list}

Advis JP, Klein I, Kuljis RO, Sarkar DK, McDonald JM, Conover CA (2003) Regulation of gonadotropin releasing hormone release by neuropeptide $Y$ at the median eminence during the preovulatory period in ewes. Neuroendocrinology $77246-257$

Allen IM, McGregor GP, Woodhams PL, Polak IM, Bloom SR (1984) Ontogeny of a novel peptide, neuropeptide $Y$ (NPY) in rat brain. Brain Res 303 197200

Asanovich KM, Johnson B, Chang WJ, Barb CR, Rampacek CB, Kraeling RR (1998) Delay of estradiol-induced surge secretion of $\mathrm{LH}$ in gilts by intracerebroventricular injection of morphine. Domest Anim Endocrinol 15 45-53

Barb CR, Chang W], Leshin LS, Rampacek GB, Kraeling RR (1994) Opioid modulation of gonadotropin releasing hormone release from the hypothalamic preoptic area in the pig. Domest Anim Endocrinol 11 375-382

Barb CR, Kraeling RR, Rampacek GB, Whisnant CS (1986) Opioid inhibition of luteinizing hormone secretion in the postpartum lactating sow. Biol Reprod 35 368371

Barraclough CA (1983) The role of catecholamines in the regulation of gonadotropin secretion. Acta Morphol Hung 31 101-115

Battmann T, Melik PS, Jeanjean B, Kerdelhue B (1991) In-vivo inhibition of the preovulatory L.H surge by substance $\mathrm{P}$ and in-vitro modulation of gonadotrophin-releasing hormone-induced $\mathrm{LH}$ release by substance $P$, oestradiol and progesterone in the female rat. J Endocrinol 130 169-175

Bauer-Dantoin AC, McDonald JK, Levine JE (1991) Neuropeptide $\mathrm{Y}$ potentiates luteinizing hormone $(\mathrm{LH})$ releasing hormone-stimulated $\mathrm{LH}$ surges in pentobarbital-blocked proestrous rats. Endocrinology 129 402-408

Bergen H, Leung PC (1986) Suppression of progesterone-induced gonadotropin surge by adrenergic agonists in estrogen-primed ovariectomized rats. Neuroendocrinology 43 397-403

Brene S, Lindefors N, Friedman W], Persson H (1990) Preprotachykinin A mRNA expression in the rat brain during development. Brain Res Dev Brain Res 57, 151-162.

Calka J (1992) Immunocytochemical localization of VIP. immunoreactive neurons in the hypothalamus of immature gilts. Neurosci Lett 136 177-180

Calka J, Majewski M, Kaleczyc J, Lakomy M (1993a)
Immunocytochemical demonstration of neuropeptide $Y$ and luteinizing hormone-releasing hormoneimmunoreactive structures in the organum vasculosum laminae terminalis of juvenile gilts. Neurosci Lett 158 21-24

Calka J, Sienkiewicz W, Majewski M, Kaleczyc J, Lakomy $M(1993 b)$ The preoptic area in the sexually immature pig. Morphological study. Folia Morphol (Warsz) $52 \quad 11-18$

Calka J, Majewski M, Kaleczyc J, Sienkiewicz W, Cozzi B, Wyrzykowski Z (1994) Distribution of neuropeptide Y-like immunoreactivity in the hypothalamic tuberal nuclei of the immature gilts. Folia Histochem Cytobiol 32 79-83

Cha $\mathrm{CI}$, Lee EY, Lee YI, Baik SH (1995) Age related change in the vasoactive intestinal polypeptide-immunoreactive neurons in the cerebral cortex of aged rats. Neurosci Lett 197 45-48

Chee CA, Roozendaal B, Swaab DF, Goudsmit E, Mirmiran $M$ (1988) Vasoactive intestinal polypeptide neuron changes in the senile rat suprachiasmatic nucleus. Neurobiol Aging 9 307-312

Clement EM, MacKinnon PC, Sheaves R (1986) Central adrenergic activity in the mediobasal hypothalamus is temporally related to surge outputs of gonadotrophins in oestrogen-stimulated infant female rats. I Endocrinol 109 45-51

Conde GL, Bicknell RJ, Herbison AE (1995) Changing patterns of Fos expression in brainstem catecholaminergic neurons during the rat oestrous cycle. Brain Res 672 68-76

Crowley WR, Kalra SP (1994) Neonatal exposure to estradiol prevents the expression of ovarian hormone-induced futeinizing hormone and prolactin surges in adulthood but not antecedent changes in neuropeptide $Y$ or adrenergic transmitter activity: implications for sexual differentiation of gonadotropin secretion. Brain Res 663 257-265

Danchin E, Dubois MP (1982) Immunocytological study of the chronology of pituitary cytogenesis in the domestic pig (Sus scrofa) with special reference to the functioning of the hypothalamo-pituitary-gonadal axis. Reprod Nutr Dev 22 135-151

Diez-Guerra FJ, Veira JA, Augood S, Emson PC (1989) Ontogeny of the novel tachykinins neurokinin $A$, neurokinin $B$ and neuropeptide $K$ in the rat central nervous system. Regul Pept 25 87-97

Duval P, Lenoir V, Garret C, Kerdelhue B (1996) Reduction of the amplitude of preovulatory $\mathrm{LH}$ and $\mathrm{FSH}$ 
surges and of the amplitude of the in vitro GnRH. induced $\mathrm{LH}$ release by substance $\mathrm{P}$. Reversal of the effect by RP 67580. Neuropharmacology 351805 1810

Elsaesser F (2001) Stimulation of porcine pituitary luteinizing hormone release by galanin: putative auto/ paracrine regulation. Neuroendocrinology 74 288299

Estrada KM, Pompolo S, Morris MJ, Tilbrook AJ, Clarke IJ (2003) Neuropeptide $Y$ (NPY) delays the oestrogen-induced luteinizing hormone (L.H) surge in the ovariectomized ewe: further evidence that NPY has a predominant negative effect on $\mathrm{LH}$ secretion in the ewe. J Neuroendocrinol 15 1011-1020

Finn PD, Clifton DK, Steiner RA (1998) The regulation of galanin gene expression in gonadotropin-releasing hormone neurons. Mol Cell Endocrinol $140137-$ 142

Gambert SR, Garthwaite TL, Pontzer CH, Hagen TC (1980) Age-related changes in central nervous system bela-endorphin and ACTH. Neuroendocrinology 31 252-255

Giorgi S, Forloni G, Baldi G, Consolo S (1995) Gene expression and in vitro release of galanin in rat hypothalamus during development. Eur I Neurosci 7 944-950

Giri M, Kaufman JM (1994) Opioidergic modulation of in vitro pulsatile gonadotropin-releasing hormone release from the isolated medial basal hypothalamus of the male guinea pig. Endocrinology 1352137. 2143

Gozes I, Schachter P, Shani Y, Giladi E (1988) Vasoactive intestinal peptide gene expression from embryos to aging rats. Neuroendocrinology $4727-31$

Graber M, Burgunder IM (1996) Ontogeny of vasoactive intestinal peptide gene expression in rat brain. Anat Embryol (Berl) 194 595-605

Gressens P (1998) Vasoactive intestinal peptide: a novel neurotrophic factor. Arch Pediatr 5 654-660

Grove KL, Brogan RS, Smith MS (2001) Novel expression of neuropeptide $Y$ (NPY) mRNA in hypothalamic regions during development: region-specific effects of maternal deprivation on NPY and Agoutirelated protein mRNA. Endocrinology 142 47714776

Gundlach AL, Burazin TC, Larm JA (2001) Distribution, regulation and role of hypothalamic galanin systems: renewed interest in a pleiotropic peptide family. Clin Exp Pharmacol Physiol 28 100-105

Hajos F, Zilles K, Gallatz K (1990) Vasoactive intestinal polypeptide (NIP) containing cells in the developing rat occipital hemisphere. Anat Embryol (Berl) 182 69-78

Hidalgo-Diaz C, Castano JP, Lopez-Pedrera R, Malagon MM, Garcia-Navarro S, Gracia-Navarro F (1998) A modulatory role for substance $P$ on the regulation of luteinizing hormone secretion by cultured porcine gonadotrophs. Biol Reprod 58 678-685

Holm IE, Geneser FA, Zimmer J (1993) Cholecystokinin-, enkephalin-, and substance P-like immunoreactivity in the dentate area, hippocampus, and sub- iculum of the domestic pig. I Comp Neurol 331310 325

Hompes PG, Vermes I, Tilders FJ, Schoemaker J (1982) Immunoreactive beta-endorphin in the hypothalamus of female rats: changes in content and release during prepubertal development. Brain Res 281281 . 286

Ichihara K, Eng I, Pond WG, Yen JT, Straus E, Yalow RS (1984) Ontogeny of immunoreactive CCK and VIP in pig brain and gut. Peptides 5 623-626

Ichihara K, Eng I, Yalow RS (1983) Ontogeny of immunoreactive CCK, VIP and secretin in rat brain and gut. Biochem Biophys Res Commun $112891-898$

Kagotani Y, Hashimoto T, Tsuruo Y, Kawano H, Daikoku S, Chihara K (1989) Development of the neuronal system containing neuropeptide $Y$ in the rat hypothalamus. Int J Dev Neurosci 7 359-374

Kalra SP (1985) Catecholamine involvement in preovulatory $\mathrm{LH}$ release: reassessment of the role of epinephrine. Neuroendocrinology 40 139-144

Kalra SP, Crowley WR (1992) Neuropeptide Y: a novel neuroendocrine peptide in the control of pituitary hormone secretion, and its relation to luteinizing hormone. Front Neuroendocrinol 13 1-46

Kalra SP, Gallo RV (1983) Effects of intraventricular administration of catecholamines on luteinizing hormone release in morphine-treated rats. Endocrinology $11323-28$

Khachaturian H, Alessi NE, Munfakh N, Watson S] (1983) Ontogeny of opioid and related peptides in the rat cns and pituitary: an immunocytochemical study. Life Sci 33 Suppl 1 61-64

Kumar MS, Becker T, Ebert K (1991) Distribution of substance $P, G n R H$, Metenkephalin in the central nervous system of the pig. Brain Res Bull 26 511-514

Lange D, Funa K, Ishisaki A, Bauer R, Wollina U (1999) Autocrine endothelial regulation in brain stem vessels of newborn piglets. Histol Histopathol 14821 . 825

Larm JA, Gundlach AL (2000) Galanin-like peptide (GALP) mRNA expression is restricted to arcuate nucleus of hypothalamus in adult male rat brain. Neuroendocrinology 72 67-71

Lieberman PB, Woods JH, Young EA (1998) The role of endogenous opioids in the luteinizing hormone surge in rats: studies with clocinnamox, a long-lasting opioid receptor antagonist. Eur J Pharmacol 35273 . 79

Lopez FJ, Meade EH, Jr., Negro-Vilar A (1993) Endogenous galanin modulates the gonadotropin and prolactin proestrous surges in the rat. Endocrinology $132795-800$

Lopez. FJ, Negro-Vilar A (1990) Galanin stimulates luteinizing hormone-releasing hormone secretion from arcuate nucleus-median eminence fragments in vitro: involvement of an alpha-adrenergic mechanism. Endocrinology 127, 2431-2436.

McDonald JK (1990) Role of neuropeptide $Y$ in reproductive function. Ann N Y Acad Sci 611 258-272

Pearson PL, Anderson LL, Jacobson CD (1996a) The prepubertal ontogeny of galanin-like immunoreactivity 
in the male Meishan pig brain. Brain Res Dev Brain Res 92 125-139

Pearson PL, Anderson LL, Jacobson CD (1996b) The prepubertal ontogeny of luteinizing hormone releasing hormone-like immunoreactivity in the diencephaIon and telencephalon of the male Meishan pig brain. Brain Res Dev Brain Res 95 157-168

Pearson PL, Anderson LL, Jacobson CD (1996c) The prepubertal ontogeny of neuropeptide Y-like immunoreactivity in the male Meishan pig brain. Brain Res Dev Brain Res 91 41-69

Pittius CW, Ellendorff F, Hollt V, Parvizi N (1987a) Ontogenetic development of proenkephalin $A$ and proenkephalin B messenger RNA in fetal pigs. Exp Brain Res 69 208-212

Pittius CW, Kley N, Loeffler JP, Hollt V (1987b) Proenkephalin $\mathbf{B}$ messenger RNA in porcine tissues: characterization, quantification, and correlation with opioid peptides. J Neurochem 48 586-592

Polkowska J, Liwska J, Dubois MP (1985) Ontogeny of neuropeptidergic systems: luteinizing hormone releasing hormone (LHRH); somatostatin (SRIF) and neurophysin (NF) in the hypothalamus of the domestic pig by immunocytochemistry. Folia Histochem Cytobiol 23 193-200

Pompei P, Severini R, Costa G, Massi M, Fattoretti P, Bertoni-Freddari C (1999) In situ hybridization analysis of preprotachykinin-A mRNA levels in young and old rats. Brain Res Mol Brain Res 64 132-136

Przybylska-Cornowicz B, Lewczuk B, Moller M (2000) immunohistochemical localization of substance $P$ in the pineal gland of the domestic pig. Folia Histochem Cytobiol 38 85-90

Rajendren G, Gibson M) (1999) Expression of galanin immunoreactivity in gonadotropin-releasing hormone neurons in mice: a confocal microscopic study. Brain Res 821 270-276

Rossmanith WG, Clifton DK, Steiner RA (1996) Galanin gene expression in hypothalamic $\mathrm{GnRH}$-containing neurons of the rat: a model for autocrine regulation. Horm Metab Res 28 257-266

Rossmanith WG, Mortola JF, Yen SS (1988) Role of endogenous opioid peptides in the initiation of the midcycle luteinizing hormone surge in normal cycling women. J Clin Endocrinol Metab 67 695-700

Ryan MC, Gundlach AL (1996) Localization of preprogalanin messenger RNA in rat brain: identification of transcripts in a subpopulation of cerebellar Purkinje cells. Neuroscience 70 709-728

Ryan MC, Loiacono RE, Gundlach AL (1997) Galanin messenger RNA during postnatal development of the rat brain: expression patterns in Purkinje cells differentiate anterior and posterior lobes of cerebellum. Neuroscience 78 1113-1127

Sahu A, Kalra SP (1992) Effects of tachykinins on luteinizing hormone release in female rats: potent inhibitory action of neuropeptide K. Endocrinology 130, 1571-1577.

Sakanaka $M$, Inagaki S, Shiosaka S, Senba E, Takagi $H$, Takatsuki K, Kawai Y, lida H, Hara Y, Tohyama M (1982) Ontogeny of substance P-containing neuron system of the rat: immunohistochemical analysis-II. Lower brain stem. Neuroscience 7 1097-1126

Sarkar DK, Fink G (1981) Gonadotropin-releasing hormone surge: possible modulation through postsynaptic alpha-adrenoreceptors and two pharmacologically distinct dopamine receptors. Endocrinology 108 862-867

Sienkiewicz W (2002) Ontogeny of the expression of some catecholamine synthesising enzymes in the female porcine preoptic area. Folia Histochem Cytobiol 40 69-76

Sienkiewicz W, Lakomy M (2003) Ontogeny of the expression of catecholamine synthesising enzymes in the female porcine median eminence arcuate nucleus complex (MEARC). Folia Morphol (Warsz) 62 239242

Sienkiewicz W, Majewski M, Kaleczyc l, Lakomy M (1996) Distribution of catecholamine-synthesizing enzymes and some neuropeptides in the median eminence-arcuate nucleus complex (MEARC) of the immature female pig. Acta Histochem $98419-434$

Singer LK, Kuper J, Brogan RS, Smith MS, Grove KL. (2000) Novel expression of hypothalamic neuropeptide $Y$ during postnatal development in the rat. Neuroreport 11 1075-1080

Sizer AR, Rokaeus A, Foster GA (1990) Analysis of the ontogeny of galanin in the rat central nervous system by immunohistochemistry and radioimmunoassay. Int J Dev Neurosci 8 81-97

Smith MJ, Jiennes L, Wise PM (2000) Localization of the VIP2 receptor protein on GnRH neurons in the female rat. Endocrinology $1414317-4320$

Tsang D, Ng SC, Ho KP, Ho WK (1982) Ontogenesis of opiate binding sites and radioimmunoassayable betaendorphin and enkephalin in regions of rat brain. Brain Res 281 257-261

Van der Beek EM, Swarts HJ, Wiegant VM (1999) Central administration of antiserum to vasoactive intestinal peptide delays and reduces luteinizing hormone and prolactin surges in ovariectomized, estrogentreated rats. Neuroendocrinology $69227-237$

Waschek JA (1995) Vasoactive intestinal peptide: an important trophic factor and developmental regulator? Dev Neurosci 17 1-7

Waschek JA, Ellison J, Bravo DT, Handley V (1996) Embryonic expression of vasoactive intestinal peptide (VIP) and VIP receptor genes. J Neurochem 66 17621765

Weick RF, Stobie KM (1992) Vasoactive intestinal peptide inhibits the steroid-induced LH surge in the ovariectomized rat. J Endocrinol 133 433-437

Weick RF, Stobie KM (1995) Role of VIP in the regulation of LH secretion in the female rat. Neurosci Biobehav.Rev 19 251-259

Woodhams PL, Allen YS, McGovern J, Allen JM, Bloom SR, Balazs R, Polak JM (1985) Immunohistochemical analysis of the early ontogeny of the neuropeptide $Y$ system in rat brain. Neuroscience 15 173-202.

Yamano $\mathrm{M}$, Inagaki S, Tateishi N, Hamaoka T, Tohyama $M$ (1984) Ontogeny of neuropeptides in the nucleus ventromedialis hypothalami of the rat: an immuno- 
histochemical analysis. Brain Res 318 253-262

Zhang L, Harlan RE (1994) Ontogeny of the distribution of tachykinins in rat cerebral cortex: immunocytochemistry and in situ hybridization histochemistry. Brain Res Dev Brain Res 77 23-36
Zilles K, Kalman M, Hajos F, Schleicher A (1991) Developmental gradients of vasoactive intestinal polypeptide (VIP)-containing neurons in the rat visual cortex detected by image analysis. Brain Res Dev Brain Res 60 137-144 